may be different underlying mechanisms of disease development between MJD and HD.

In conclusion, MJD has three factors that influence the age of the onset. One is the length of CAG repeats of the $M J D 1$ gene, another is gender, and third is dosage of the gene with expanded CAG repeats. The effects of the last two factors suggest that the expanded CAG repeats in
MJD may cause the disease in a manner different from other CAG repeat diseases.

Hideshi Kawakami

Hirofumi Maruyama

Shigenobu Nakamura

Third Department of Internal Medicine, Hiroshima University, School of Medicine, Hiroshima 734, Japan
Yoshiya Kawaguchi

Akira Kakizuka

Department of Pharmacology, Kyoto University, Faculty of Medicine, Kyoto 606-01, Japan

\section{Manabu Doyu}

Gen Sobue

Fourth Department of Internal Medicine, Aichi Medical University, Nagakute, Aichi 480-11, Japan

\title{
Anastasia and Anna Anderson
}

Sir - I would like to point out that many of the statements appearing in the editorial in the November 1994 issue of Nature Genetics ('Anastasia and the tools of justice', 8, 205-206; 1994), are wrong. If your journal is truly part of the 'scientific community' and devoted to factual accuracy, those errors deserve acknowledgement and correction.

You wrongly state that I 'refuse to accept' the scientific results achieved by Dr. Peter Gill and colleagues ${ }^{1}$. That is absolutely untrue. We have never questioned their scientific results. We instigated their cooperation and full sharing of information, and continue our efforts toward that end. Nevertheless, we do notblithely accept the 'conclusions' and inferences that are being drawn from the various scientific results and the subsequent comparisons.

Your assertions that (i) Dr. Gill 'in fact' said Anna Anderson was most likely a Polish farm worker, and (ii) two independent groups arrived at the 'same conclusion', are not accurate. Dr. Gill made no such statement [at the press conference held last year to announce his group's results]. He carefully confined himself to his assessment of the tissue tested, a term he used repeatedly. Indeed, in your correspondence from Gill et al. (cited above), he carefully refers to 'putative Anna Anderson samples' (emphasis added).

You also mention the work of Dr. Bernd Hermann as arriving at the same conclusion ('she was most likely a Polish farm worker'), which is not true. In fact, the simple tandem repeats (STRs) from the blood slides you cited did not match those derived by the Forensic Science Service from the tissue samples held in Charlottesville. We are following closely the continuing efforts for comparison of any miDNA from those slides. So far, there has been no match to maternally descended Schanzkowska mtDNA ${ }^{1}$. The discrepancies in the STRs are so extensive, as we understood from $\mathrm{Dr}$. Gill, that the two sources could not likely have come from the same person.

Mr. Syd Mandelbaum, who is preparing a text on the subject of DNA as legal evidence, arranged for Dr. Stoneking's work on the hair sample. There is no certain provenance or chain of custody for the source of the hair. Dr. Stoneking's findings were simply that the mtDNA profile from the hair matched that derived by Gill's group from the blood tissue of Karl Maucher'. At our last contact, Dr. Stoneking had not derived any STRs from that follicular hair, nor did he determine the sex of the mtDNA source.

The rational human experiences of persons who actually knew the individual called Anna Anderson, and of those who know the other evidence of her identity, are in direct conflict with the conclusion or inference drawn that this specific individual could possibly have been a Polish peasant of that era. When inferences drawn from science conflict with rational human experience, that conflict should be resolved. That is the task we are continuing.

\section{R. Richard Schweitzer}

Attorney at Law,

746 Walker Road, P.O, Box 788, Great Falls, Virginia 22066, USA

1. Gill, P. et al. Nature Genet. 9, 9-10 (1995). 